PROCEEDINGS OF THE

AMERICAN MATHEMATICAL SOCIETY

Volume 137, Number 6, June 2009, Pages 1907-1913

S 0002-9939(09)09672-5

Article electronically published on January 15, 2009

\title{
THE GHOST DIMENSION OF A RING
}

\author{
MARK HOVEY AND KEIR LOCKRIDGE
}

(Communicated by Birge Huisgen-Zimmermann)

\begin{abstract}
We introduce the concept of the ghost dimension gh.dim. $R$ of a ring $R$. This is the longest nontrivial chain of maps in the derived category emanating from a perfect complex such that each map is zero on homology. We show that w.dim. $R \leq$ gh.dim. $R$ with equality if $R$ is coherent or w.dim. $R=1$.
\end{abstract}

\section{INTRODUCTION}

Given a ring $R$, one can study $R$ by looking at properties of elements of $R$, properties of the category $R$-mod of right $R$-modules, or properties of its unbounded derived category $\mathcal{D}(R)$. If we allow ourselves to remember the object $R$ (thus ruling out Morita and derived equivalences), these approaches all contain the same information. However, there are generalized rings where not all of these approaches are available. In algebraic topology, for example, there are structured ring spectra $E$ (often called $S$-algebras or symmetric ring spectra). For such an $E$, there are no elements, and there is no abelian category of $E$-modules. But there is a triangulated category $\mathcal{D}(E)$ analogous to $\mathcal{D}(R)$. If one wants to define various homological dimensions of $E$, then one needs to look at how to define homological dimensions of $R$ solely in terms of the derived category $\mathcal{D}(R)$ (with its distinguished object $R$ ). In this paper, we describe how to recover the right global dimension of $R$ from $\mathcal{D}(R)$ and its distinguished object $R$, and we introduce another invariant, the ghost dimension of $R$, that we originally thought would be the weak dimension or the finitistic right global dimension of $R$. However, we now believe this to be a new invariant of $R$.

In more detail, given a ring $R$, let $\mathcal{D}(R)$ denote the unbounded derived category of right $R$-modules. A map $f: X \rightarrow Y$ in $\mathcal{D}(R)$ is called a ghost if $H_{*} f=0$. (Note that to define a ghost, we need to remember the object $R$, since $H_{*} X=$ $\mathcal{D}(R)\left(\Sigma^{*} R, X\right)$, so ghosts are not derived invariant.) A complex $X$ is said to have ghost length $n$, written gh. len. $X=n$, if every composite

$$
X \stackrel{f_{1}}{\longrightarrow} Y_{1} \stackrel{f_{2}}{\longrightarrow} \cdots \stackrel{f_{n+1}}{\longrightarrow} Y_{n+1}
$$

of $n+1$ ghosts is 0 in $\mathcal{D}(R)$, and there exists a composite of $n$ ghosts from $X$ that is not 0 in $\mathcal{D}(R)$. Since the complex $R$ itself has ghost length 0 , one might think that the ghost length of a complex is analogous to the projective dimension of a module. Hence, the maximal ghost length should be the right global dimension of $R$. This is correct, and we prove it in Theorem 1.5, following the second author's

Received by the editors November 30, 2007, and, in revised form, June 10, 2008.

2000 Mathematics Subject Classification. Primary 16E10; Secondary 18G20, 13D05, 55P43.

(C)2009 American Mathematical Society
Reverts to public domain 28 years from publication 1907 
thesis [Loc06, Theorem 4.4.4]. Now a finitely presented module is analogous to a perfect complex in $\mathcal{D}(R)$, so the maximal ghost length of a perfect complex should be related to the maximal projective dimension of a finitely presented module, or perhaps to the weak dimension of $R$ (since these two invariants are equal when $R$ is coherent). However, we have not been able to prove this, and so we define the ghost dimension of $R$, written gh. $\operatorname{dim} . R$, to be the maximum ghost length of any perfect complex in $\mathcal{D}(R)$ (or $\infty$ if there is no such maximum).

The authors and G. Puninski proved that ghost dimension 0 is equivalent to weak dimension 0 (i.e., von Neumann regular) in [HLP07, although the authors referred to $R$ having ghost dimension 0 as $R$ satisfying the strong generating hypothesis, based on analogy with the stable homotopy category in algebraic topology. In the present paper, we prove that w. $\operatorname{dim} . R \leq \operatorname{gh}$. $\operatorname{dim} . R$, with equality holding if $R$ is right coherent or w. dim. $R \leq 1$. We have not been able to resolve the general case, though we suspect there is a counterexample.

The ghost dimension in the stable module category of a finite group has been studied in [CCM08].

\section{Global dimension}

The object of this section is to prove that r. gl. dim. $R$ is the maximum ghost length of a complex in $\mathcal{D}(R)$. Along the way, we prove some basic facts about ghost length that we will need later.

We first recall that the ghost maps $\mathcal{I}$ are part of a projective class $(\mathcal{P}, \mathcal{I})$ in $\mathcal{D}(R)$ Chr98, Section 8]. Here $\mathcal{P}$ is the collection of all complexes isomorphic in $\mathcal{D}(R)$ to a complex of projectives with zero differential. To say that $(\mathcal{P}, \mathcal{I})$ is a projective class means three things:

(1) $f: X \rightarrow Y \in \mathcal{I}$ if and only if the composite $f g$ is 0 for all $g: P \rightarrow X$ with $P \in \mathcal{P}$

(2) $P \in \mathcal{P}$ if and only if $f g=0$ for all $f: X \rightarrow Y \in \mathcal{I}$ and all $g: P \rightarrow X$;

(3) for all $X \in \mathcal{D}(R)$ there is a cofiber sequence

$$
P \rightarrow X \stackrel{f}{\rightarrow} Y \rightarrow \Sigma P
$$

with $P \in \mathcal{P}$ and $f \in \mathcal{I}$.

These conditions are all easy to check for the ghost projective class. For example, given $X$, choose a free module $P_{n}$ mapping onto $H_{n} X$ for all $n$, and let $P=$ $\bigoplus_{n} S^{n} P_{n}$, where $S^{n} P_{n}$ is the complex consisting of $P_{n}$ concentrated in degree $n$. There is an obvious map $S^{n} P_{n} \rightarrow X$ that is onto in homology, from which it follows that the cofiber $X \rightarrow Y$ is a ghost.

As Christensen describes in [Chr98, Theorem 3.5], there is an induced projective class $\left(\mathcal{P}_{n+1}, \mathcal{I}^{n+1}\right)$, where $\mathcal{I}^{n+1}$ consists of the $(n+1)$-fold composites of ghost maps, and so, by definition, $\mathcal{P}_{n+1}$ is the collection of complexes of ghost dimension $\leq n$.

In particular, from Christensen's work we deduce the following proposition.

Proposition 1.1. Let $R$ be a ring.

(1) A complex $X \in \mathcal{D}(R)$ has gh. len. $X \leq n$ if and only if there is an exact triangle

$$
Z \rightarrow X^{\prime} \rightarrow P \rightarrow \Sigma Z
$$


in $\mathcal{D}(R)$, where $X$ is a summand in $X^{\prime}$, gh. len. $Z \leq n-1$ and gh. len. $P=0$, and $\Sigma$ is the shift functor.

(2) $X$ has finite ghost length if and only if it is in the thick subcategory generated by the complexes of projective modules with zero differential.

Recall that a full subcategory $\mathcal{C}$ is thick if it is closed under shifts and summands, and if two out of three objects in an exact triangle are in $\mathcal{C}$, so is the third (where we view an object and its shift as the same). Note also that this means that all perfect complexes have finite ghost length, since they are in the thick subcategory generated by the complex $R$.

Proof. The first statement follows from [Chr98, Theorem 3.5] and the discussion immediately preceding it. For the second statement, note first that complexes of projective modules with zero differential have ghost length 0, so [Chr98, Note 3.6] implies that everything in the thick subcategory generated by them has finite ghost length. For the converse, we proceed by induction on the ghost length of $X$. The induction step follows from part (1). The base case is clear, since every complex of ghost length 0 is a complex of projectives with zero differential.

Corollary 1.2. Suppose $X$ is a complex of projectives such that $X_{i}=0$ for all but $n+1$ values of $i$. Then gh. len. $X \leq n$.

Proof. Use induction and part (1) of Proposition 1.1.

Christensen's work also gives us the following proposition, which is a restatement of [Chr98, Theorem 8.3].

Proposition 1.3. Suppose r. gl. $\operatorname{dim} . R \leq n$. Then every complex $X$ in $\mathcal{D}(R)$ has gh. len. $X \leq n$.

One way to think about this proposition is in terms of the spectral sequence

$$
E_{2}^{s, t}=\operatorname{Ext}_{R}^{s, t-s}\left(H_{*} X, H_{*} Y\right) \Rightarrow \mathcal{D}(R)(X, Y)_{s} .
$$

This is sometimes known as the hypercohomology spectral sequence in algebra, and the universal coefficient spectral sequence in topology. It is conditionally convergent, and the associated filtration on $\mathcal{D}(R)(X, Y)$ is the ghost filtration. This spectral sequence is described in [Chr98, Section 4] for a general projective class, but is well known in this case. In any case, if the right global dimension of $R$ is $\leq n$, then this spectral sequence has only $n+1$ nonzero rows at $E_{2}$, so of course also at $E_{\infty}$, so the ghost length is at most $n$.

To prove the converse of this proposition, we note one simple source of ghosts. For any complex $X$, we can let $X[m, \infty]$ be the complex with $X[m, \infty]_{i}=X_{i}$ if $i \geq m$ and 0 if $i<m$, with the nonzero differentials coinciding with those of $X$. Then there is an obvious map $X \rightarrow X[m, \infty]$ of complexes, and this map will be a ghost if $X$ has no homology in degrees $\geq m$. In particular, we get the following lemma.

Lemma 1.4. If $X$ is the projective resolution of a module $M$, then gh. len. $X=$ proj. $\operatorname{dim} . M$. In particular, gh. $\operatorname{dim} . R$ is at least as big as the maximal finite projective dimension of an $F P_{\infty}$-module. 
Recall that an $F P_{\infty}$-module is a module which has a projective resolution by finitely generated projectives. If the $F P_{\infty}$-module $M$ has finite projective dimension, then there is a projective resolution of $M$ that is a perfect complex, so the second statement of the lemma follows from the first.

Proof. Write $X$ as the sequence

$$
\cdots \stackrel{d_{k+1}}{\longrightarrow} X_{k} \stackrel{d_{k}}{\longrightarrow} X_{k-1} \stackrel{d_{k-1}}{\longrightarrow} \cdots \stackrel{d_{1}}{\longrightarrow} X_{0} .
$$

Suppose proj. $\operatorname{dim} . M=n$, so that $X_{k}=0$ for $k>n$. Then Corollary 1.2 implies gh. len. $X \leq n$. Now, the sequence of ghosts

$$
X \rightarrow X[1, \infty] \rightarrow X[2, \infty] \rightarrow \cdots \rightarrow X[n, \infty]
$$

cannot be 0 in $\mathcal{D}(R)$, since if it were it would be chain homotopic to 0 , and so null homotopic, so that $d_{n}: X_{n} \rightarrow X_{n-1}$ would be a split monomorphism. This would mean that the kernel of $d_{n-2}$ is projective, so that proj. $\operatorname{dim} . M \leq n-1$. Hence gh. len. $X \geq n$ (even if $n=\infty)$.

This lemma together with Proposition 1.3 gives us the following theorem, due to the second author [Loc06, Theorem 4.4.4].

Theorem 1.5. The maximum ghost length of an object in $\mathcal{D}(R)$ is r.gl. dim. $R$. Hence gh. dim. $R \leq$ r. gl. $\operatorname{dim} . R$.

\section{WEAK DIMENSION}

The object of this section is to show that gh. $\operatorname{dim} . R \geq$ w. $\operatorname{dim} . R$, with equality holding when $R$ is right coherent or when w. $\operatorname{dim} . R \leq 1$.

Theorem 2.1. Suppose $R$ is a ring. Then w. dim. $R \leq \operatorname{gh}$. dim. $R$.

Proof. Let $n=$ gh. $\operatorname{dim}$. $R$, which we can of course assume is finite. Let $M$ be an $R$-module, and let

$$
\cdots \stackrel{d_{k+1}}{\longrightarrow} P_{k} \stackrel{d_{k}}{\longrightarrow} P_{k-1} \stackrel{d_{k-1}}{\longrightarrow} \cdots \stackrel{d_{1}}{\longrightarrow} P_{0} \stackrel{d_{0}}{\longrightarrow} M
$$

be a free resolution of $M$. Let $M_{k+1}$ be the kernel of $d_{k}$, so we have short exact sequences

$$
0 \rightarrow M_{k+1} \stackrel{i_{k+1}}{\longrightarrow} P_{k} \stackrel{q_{k}}{\longrightarrow} M_{k} \rightarrow 0
$$

with $M_{0}=M$ and $i_{k} q_{k}=d_{k}$. We need to show that $M_{n}$ is flat, which we will do by applying [Lam99, Theorem 4.23] to the short exact sequence

$$
0 \rightarrow M_{n+1} \rightarrow P_{n} \rightarrow M_{n} \rightarrow 0
$$

So take a $c \in M_{n+1}$. To use [Lam99, Theorem 4.23], we need to construct a map $P_{n} \rightarrow M_{n+1}$ that takes $c$ to itself. We first build a perfect complex $Q$ that contains c. Begin by choosing a $b \in P_{n+1}$ with $d_{n+1} b=c$, and let $Q_{n+1}=R$. Now find a finitely generated summand $Q_{n}$ of $P_{n}$ that contains $c$, and let $d: Q_{n+1} \rightarrow Q_{n}$ take 1 to $c$. Then $d\left(Q_{n}\right)$ lies in a finitely generated summand $Q_{n-1}$ of $P_{n-1}$, so, since $d c=0$, we can define $d$ on $Q_{n}$ to be the restriction of $d$ on $P_{n}$. Continuing in this fashion, we get a perfect complex $Q$ and a map $Q \rightarrow P$ which takes $1 \in Q_{n+1}$ to $b$, and is the inclusion $Q_{k} \rightarrow P_{k}$ for all $k \leq n$.

Now consider the sequence of ghosts

$$
Q \rightarrow P[1, \infty] \rightarrow P[2, \infty] \rightarrow \cdots \rightarrow P[n+1, \infty]
$$


where the first map is the composite $Q \stackrel{j}{\rightarrow} P \rightarrow P[1, \infty]$. Since gh. len. $Q \leq n$, this composite of $n+1$ ghosts is nullhomotopic. Such a null chain homotopy gives us a map $D: Q_{n} \rightarrow P_{n+1}$ and an element $x \in P_{n+2}$ such that $d_{n+2} x+D c=b$. In particular, $d_{n+1} D c=c$. Consider the map

$$
P_{n} \stackrel{\tau}{\rightarrow} Q_{n} \stackrel{D}{\longrightarrow} P_{n+1} \stackrel{q_{n+1}}{\longrightarrow} M_{n+1},
$$

where $\tau$ is a splitting of the inclusion $Q_{n} \rightarrow P_{n}$. Then

$$
q_{n+1} D \tau(c)=d_{n+1} D c=c,
$$

as required to apply [Lam99, Theorem 4.23].

We have not been able to prove that $\operatorname{gh} . \operatorname{dim} . R=\mathrm{w}$. $\operatorname{dim} . R$ for a general ring $R$. For right coherent $R$, however, this is true.

Proposition 2.2. Suppose $R$ is right coherent. Then $\operatorname{gh} . \operatorname{dim} . R=$ w. $\operatorname{dim} . R$.

Proof. We will show that in the spectral sequence

$$
E_{2}^{s, t}=\operatorname{Ext}_{R}^{s, t-s}\left(H_{*} X, H_{*} Y\right) \Rightarrow \mathcal{D}(R)(X, Y)_{s}
$$

discussed after Proposition 1.3. $E_{2}$ vanishes for $s>$ w. $\operatorname{dim} . R$ if $X$ is perfect. Hence the same will be true for $E_{\infty}$. Since $E_{\infty}^{s, *}$ represents the maps that are $s$-fold composites of ghost maps but not $(s-1)$-fold such composites, we conclude that every nonzero map is a composite of at most w. dim. $R$ ghosts, giving us the desired result.

First note that because $R$ is right coherent, finitely presented right modules are closed under kernels and cokernels. Thus if $X$ is a perfect complex, then $H_{i} X$ is a finitely presented $R$-module for all $i$. On the other hand, again because $R$ is right coherent, proj. $\operatorname{dim} . M$ is equal to the flat dimension of $M$ for all finitely presented $M$. Indeed, because $R$ is right coherent, there is a projective resolution

$$
\cdots \rightarrow P_{k} \rightarrow P_{k-1} \rightarrow \cdots \rightarrow P_{0} \rightarrow M
$$

of $M$ in which each $P_{k}$ is finitely generated projective. If the flat dimension of $M$ is $n$, then the kernel of $P_{n-1} \rightarrow P_{n-2}$ is flat. But it is also finitely presented, again since $R$ is right coherent. So it is projective, and so proj. $\operatorname{dim} . M \leq n$. Hence we have proj. $\operatorname{dim} . M \leq \mathrm{w}$. $\operatorname{dim} . R$ for all finitely presented $M$, completing the proof.

We can also prove gh. $\operatorname{dim} . R=$ w. $\operatorname{dim} . R$ when w. $\operatorname{dim} . R=1$. To prove this, we need the following slight generalization of the theorem of Villamayor Lam99, Theorem 4.23] that we have already used in the proof of Theorem 2.1.

Lemma 2.3. Suppose we have a short exact sequence of right modules over a ring $R$,

$$
0 \rightarrow K \rightarrow P \rightarrow M \rightarrow 0,
$$

where $P$ is projective. Then the following conditions are equivalent.

(1) $M$ is flat.

(2) For every finitely generated submodule $L$ of $K$, there is a map $\theta: P \rightarrow K$ such that $\theta(x)=x$ for all $x \in L$.

(3) For every element $c$ of $K$, there is a map $\theta: P \rightarrow K$ such that $\theta(c)=c$. 
Proof. Find a $Q$ such that $P \oplus Q$ is free. Then we have a short exact sequence

$$
0 \rightarrow K \oplus Q \rightarrow P \oplus Q \rightarrow M \rightarrow 0
$$

We can now apply Lam99, Theorem 4.23]. More precisely, suppose that $M$ is flat and $L$ is a finitely generated submodule of $K$. Then, by [Lam99, Theorem 4.23], there is a map $f: P \oplus Q \rightarrow K \oplus Q$ such that $f(x, 0)=(x, 0)$ for all $x \in L$. But then we can define $\theta$ to be the composite

$$
P \rightarrow P \oplus Q \stackrel{f}{\rightarrow} K \oplus Q \rightarrow K
$$

to see that $(1) \Rightarrow(2)$. It is obvious that $(2) \Rightarrow(3)$. To see that $(3) \Rightarrow(1)$, take an element $(c, q)$ of $K \oplus Q$. By assumption, there is a map $\theta: P \rightarrow K$ such that $\theta(c)=c$. Define $f: P \oplus Q \rightarrow K \oplus Q$ by $f(x, y)=(\theta(x), y)$. Then $f(c, q)=(c, q)$. So [Lam99, Theorem 4.23] implies that $M$ is flat.

We can use this improvement to characterize rings of weak dimension 1.

Proposition 2.4. A ring $R$ has w. $\operatorname{dim} . R \leq 1$ if and only if, for every diagram

$$
P_{2} \stackrel{d_{2}}{\longrightarrow} P_{1} \stackrel{d_{1}}{\longrightarrow} P_{0}
$$

of finitely generated projectives with $d_{1} d_{2}=0$, there is a map $f: P_{1} \rightarrow P_{1}$ with $d_{1} f=0$ and $f d_{2}=d_{2}$.

Proof. Suppose $R$ has weak dimension $\leq 1$, and consider the short exact sequence

$$
0 \rightarrow \operatorname{ker} d_{1} \rightarrow P_{1} \rightarrow \operatorname{im} d_{1} \rightarrow 0 \text {. }
$$

Since $R$ has weak dimension $\leq 1, \operatorname{im} d_{1} \subseteq P_{0}$ is flat. Apply Lemma 2.3 to the finitely generated submodule im $d_{2}$ of ker $d_{1}$ to get a map $f: P_{1} \rightarrow \operatorname{ker} d_{1}$ such that $f(x)=x$ for all $x \in \operatorname{im} d_{2}$. This means that $d_{1} f=0$ and $f d_{2}=d_{2}$, as required.

For the converse, it suffices to show that $\operatorname{Tor}_{2}^{R}(N,-)=0$ for all finitely presented $N$. Take a finite presentation

$$
P_{1} \stackrel{d_{1}}{\longrightarrow} P_{0} \rightarrow N \rightarrow 0
$$

of $N$, where $P_{1}$ and $P_{0}$ are free and finitely generated. This gives us the short exact sequence

$$
0 \rightarrow K \rightarrow P_{1} \rightarrow \operatorname{im} d_{1} \rightarrow 0 .
$$

We need to show that $\operatorname{im} d_{1}$ is flat. Using Lam99, Theorem 4.23], it suffices to show that for any $c \in K$, there is a map $f: P_{1} \rightarrow K$ such that $f(c)=c$. But $c$ then gives us the complex

$$
R \stackrel{g}{\rightarrow} P_{1} \stackrel{d_{1}}{\longrightarrow} P_{0}
$$

of finitely generated projectives, where $g(1)=c$. By hypothesis, then, there is a map $f: P_{1} \rightarrow K$ such that $f(c)=c$.

Theorem 2.5. If w. dim. $R \leq 1$, then $\operatorname{gh} . \operatorname{dim} . R=$ w. $\operatorname{dim} . R$.

Proof. If w. $\operatorname{dim} . R=0$, this is proved in [HLP07, Theorem 1.3]. Thus, in view of Theorem 2.1, we must show that w. $\operatorname{dim} . R=1 \mathrm{implies} g h . \operatorname{dim} . R \leq 1$. We do this by showing that every perfect complex is a retract of a direct sum of complexes with only two nonzero entries. In view of Corollary 1.2, this will complete the proof.

Suppose $P$ is a perfect complex, with maps $d_{n}: P_{n} \rightarrow P_{n-1}$. Since $d_{n} d_{n+1}=0$, Proposition 2.4 implies that there are maps $f_{n}: P_{n} \rightarrow$ ker $d_{n}$ such that $f_{n} d_{n+1}=$ $d_{n+1}$. For each $n$, choose a surjection $q_{n}: Q_{n} \rightarrow \operatorname{ker} d_{n}$ where $Q_{n}$ is projective, 
and choose a lift $g_{n}: P_{n} \rightarrow Q_{n}$ so that $q_{n} g_{n}=f_{n}$. Now define a two-term chain complex $T^{n}$ with $T_{n}^{n}=P_{n}$ and $T_{n-1}^{n}=Q_{n-1}$, with differential $g_{n-1} d_{n}$.

We claim that there are chain maps

$$
P \stackrel{\phi}{\rightarrow} \bigoplus_{k} T^{k} \stackrel{\psi}{\rightarrow} P
$$

exhibiting $P$ as a retract of $\bigoplus_{k} T^{k}$. Note that the $n$th term of the complex $\bigoplus_{k} T^{k}$ is $Q_{n} \oplus P_{n}$, with differential $D_{n}(x, y)=\left(g_{n-1} d_{n} y, 0\right)$. Define $\psi_{n}: Q_{n} \oplus P_{n} \rightarrow P_{n}$ by $\psi_{n}(x, y)=q_{n}(x)+y$. Then

$$
d_{n} \psi_{n}(x, y)=d_{n} y
$$

and

$$
\psi_{n-1} D_{n}(x, y)=\psi_{n-1}\left(g_{n-1} d_{n} y, 0\right)=q_{n-1} g_{n-1} d_{n} y=f_{n-1} d_{n} y=d_{n} y,
$$

so $\psi$ is a chain map.

Now define $\phi_{n}: P_{n} \rightarrow Q_{n} \oplus P_{n}$ by $\phi_{n}(x)=\left(g_{n}(x), x-f_{n} x\right)$. Then

$$
D_{n} \phi_{n}(x)=\left(g_{n-1} d_{n}\left(x-f_{n} x\right), 0\right)=\left(g_{n-1} d_{n} x, 0\right)
$$

and

$$
\phi_{n-1} d_{n}(x)=\left(g_{n-1} d_{n} x, d_{n} x-f_{n-1} d_{n} x\right)=\left(g_{n-1} d_{n} x, 0\right) .
$$

Thus $\phi$ is a chain map, and one can readily verify that

$$
\psi_{n} \phi_{n}(x)=\psi_{n}\left(g_{n} x, x-f_{n} x\right)=q_{n} g_{n} x+x-f_{n} x=x,
$$

showing that $P$ is a retract of $\bigoplus_{n} T^{n}$.

\section{REFERENCES}

[CCM08] Sunil K. Chebolu, J. Daniel Christensen, and Ján Mináč, Ghosts in modular representation theory, Adv. Math. 217 (2008), 2782-2799. MR2397466 (2008m:20018)

[Chr98] J. Daniel Christensen, Ideals in triangulated categories: Phantoms, ghosts and skeleta, Adv. Math. 136 (1998), no. 2, 284-339. MR:1626856 (99g:18007)

[HLP07] Mark Hovey, Keir Lockridge, and Gena Puninski, The generating hypothesis in the derived category of a ring, Math. Z. 256 (2007), no. 4, 789-800. MR2308891

[Lam99] T. Y. Lam, Lectures on modules and rings, Graduate Texts in Mathematics, vol. 189, Springer-Verlag, New York, 1999. MR.1653294 (99i:16001)

[Loc06] Keir H. Lockridge, The generating hypothesis in general stable homotopy categories, Ph.D. thesis, University of Washington, 2006.

Department of Mathematics, Wesleyan University, Middletown, Connecticut 06459

E-mail address: hovey@member.ams.org

Department of Mathematics, Wake Forest University, Winston-Salem, North CaroLINA 27109

E-mail address: lockrikh@wfu.edu 